\title{
In vivo quantification of nanoparticle association with immune cell subsets in blood
}

Yih Rue Ong, Robert De Rose, Angus Johnston*
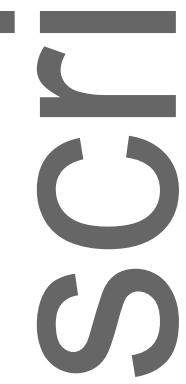

((Optional Dedication))

Y. R. Ong, Dr. R. De Rose, Dr. A. Johnston

1) Monash Institute of Pharmaceutical Sciences, Monash University, Parkville, VIC, Australia.

2) ARC Centre of Excellence in Convergent Bio-Nano Science and Technology, Monash University,

Parkville, VIC, Australia.

E-mail: angus.johnston@monash.edu

Keywords: immunology, nanoparticle, blood association, ex vivo, in vivo

Nanoparticles offer great promise for more effective drug delivery. However, their particulate nature typically results in rapid systemic clearance by immune cells in blood. Currently, to understand these interactions, nanoparticle association is probed ex vivo with whole blood. While ex vivo assays give important information about the relative cell association, they do not consider changes in immune cell homeostasis or the complex mixing behavior that occurs in vivo. To address this, we developed a Nanoparticle In Vivo Immune-cell Association (NIVIA) assay to study the in vivo association of unmodified and PEGylated liposomes with immune cells, and compared this to the ex vivo association in static whole blood. In vivo, we observed that neutrophils play a significantly greater role in nanoparticle binding than suggested by ex vivo assays. The increased influence of neutrophils in vivo was largely due to a significant increase in number of circulating neutrophils after intravenous injection. Conversely, the number of circulating monocytes significantly decreased after intravenous injection, leading to significantly less total association of liposomes to monocytes compared to ex vivo. This novel in vivo immune cell binding assay sheds new light on the fate of nanoparticles following intravenous delivery.

This is the author manuscript accepted for publication and has undergone full peer review but has not been through the copyediting, typesetting, pagination and proofreading process, which may lead to differences between this version and the Version of Record. Please cite this article as doi: 10.1002/adhm.202002160.

This article is protected by copyright. All rights reserved. 


\section{Introduction}

Nanoparticles are increasingly being used for medical applications as carriers to transport drugs to sites of interest (e.g. tumor), for diagnostic purposes and for vaccine applications. ${ }^{[1-3]}$ For many applications, nanoparticles will be administered intravenously. ${ }^{[4]}$ A significant impediment for intravenous delivery is that nanoparticles are readily identified as foreign bodies by the innate immune system and can undergo rapid systemic clearance. Exposure to blood results in plasma proteins adsorbing on the surface of the nanoparticles, referred to as the protein corona, which modifies the physicochemical properties of the nanoparticle and opsonizes the particles for phagocytosis and removal from circulation. ${ }^{[5]}$ The role of the protein corona has been studied by incubating nanoparticles in solutions containing multiple protein components from serum and plasma. ${ }^{[6,7]}$ Modifications to the surface of the nanoparticles, including the low-fouling hydrophilic polymer poly(ethylene glycol) (PEG), are commonly used to limit opsonization and improve delivery to the site of action. ${ }^{[8]}$ However, it is hard to predict how these modifications modulate protein interactions, as protein adsorption is governed by density, length and confirmation of the surface coating, as well as the properties of the underlying nanoparticle. Therefore, it is important to investigate how nanoparticles, and any modifications made to them, influence binding to cells.

In vitro assays have been used to assess the phagocytic ability of cultured macrophages or dendritic cells following nanoparticle exposure. ${ }^{[9,10]}$ However, these in vitro studies do not account for all plasma proteins nor the many different types of cells in blood. Blood is composed of erythrocytes, platelets, leukocytes and plasma. Capture of nanoparticles typically occurs by leukocytes through phagocytic and non-phagocytic mechanisms. In blood, the phagocytic cells are monocytes, neutrophils, eosinophils and dendritic cells that, on a per cell basis, have the greatest capacity to This article is protected by copyright. All rights reserved. 


\section{WILEY-VCH}

remove nanoparticles from circulation. Nanoparticles can also adhere to B cells, T cells and NK cells that make up the pool of lymphocytes, but these cells have a comparatively limited capacity to internalize particles, which theoretically reduces their role in nanoparticle capture. ${ }^{[11]}$ By incubating nanoparticles in whole blood, the complex interaction of nanoparticles with all the components of blood are taken into consideration. Currently, the gold standard for evaluation of nanoparticle interactions with blood is an ex vivo whole blood assay.

The ex vivo blood assay is a quick and simple surrogate for evaluating the initial complex interplay of nanoparticle interactions with plasma protein and cells following intravenous injection. ${ }^{[12]}$ Nanoparticles are also subjected to complex mixing within blood vessels that changes as the particles move successively through arteries with high velocity flow, to capillaries within tissues where flow is dramatically reduced and then into veins. The influence of all these factors on nanoparticle sequestration in blood can therefore only be captured by evaluating the cellular interactions in vivo.

A limited number of preclinical in vivo studies have quantified the association of nanoparticles with immune cells in blood through flow cytometry, but these studies only looked at interactions with specific subsets of cells. ${ }^{[13-15]}$ These were principally performed with antibodies against the common leukocyte antigen CD45, or a single immune cell subset of interest (e.g. neutrophils). In this study, we expanded upon the current understanding of nanoparticle-immune cell interactions in the blood by developing a Nanoparticle In Vivo Immune-cell Association (NIVIA) assay to probe nanoparticle interactions with all immune cell subsets in vivo, and directly compared these interactions to nanoparticles incubated with whole blood ex vivo. Unmodified phosphatidylcholine liposomes and PEGylated phosphatidylcholine liposomes, which have similar formulations to clinical therapies (Myocet and Doxil respectively, but without the doxorubicin) were used in this evaluation. 


\section{Results}

To probe for differences between in vivo and ex vivo nanoparticle-immune cell interactions, we compared nanoparticle association with immune cells in blood following in vivo administration to mice (NIVIA), to nanoparticles incubated in whole mouse blood ex vivo. Immune cells were identified by labeling with a cocktail of antibodies (Table S1). The two types of phosphatidylcholine (PC) liposomes used in this study - unmodified liposomes (referred to from herein as PC liposomes) and PC liposomes modified with $\sim 5$ mol \% $2000 \mathrm{MW}$ polyethylene glycol (PEG-liposomes) are similar to clinical liposomal formulations Myocet and Doxil respectively. Both types of liposomes were fluorescently labeled with a lipophilic dye, DiD, that inserts into the liposome membrane. ${ }^{[16]}$ The mean $( \pm S D$ ) particle sizes were $116 \pm 26 \mathrm{~nm}$ for PC liposomes and $127 \pm 30 \mathrm{~nm}$ for PEG-liposomes (Figure S1). The zeta potential for PC liposomes $(-7 \pm 9 \mathrm{mV})$ and PEG-liposomes $(-11 \pm 7 \mathrm{mV})$ were similar. The negative charge was expected due to the presence of a small amount of the negatively charged 1,2-Distearoyl-sn-Glycero-3-Phosphoethanolamine (DSPE) in each formulation.

To match the concentration of liposomes to immune cells in the ex vivo and in vivo experiments, the ex vivo dose of liposomes was calculated based on the assumption that the total blood volume was $7 \%$ of the body weight of the mouse $(70 \mathrm{~mL} / \mathrm{kg}){ }^{[17]}$ The experimental steps for the NIVIA and ex vivo blood assay are outlined in Figure 1. Liposome interactions with immune cells were quantified using flow cytometry by evaluating the DiD signal associated with different immune cell subsets (Figure S2). The fluorescence intensity of PEG-liposomes was 59\% brighter than unmodified liposomes (Figure S3a). To take this into account, the MFI of the PC liposomes was multiplied by 1.59. The endotoxin level of all liposome batches and PBS used in this study was indicated by manufacturers to be $<0.1 \mathrm{EU} / \mathrm{mg}$ and therefore comparable to levels in approved human therapies.

This article is protected by copyright. All rights reserved. 


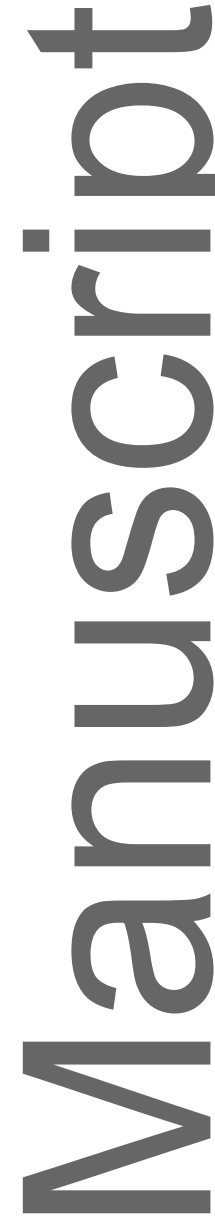

HIVA assay

d)
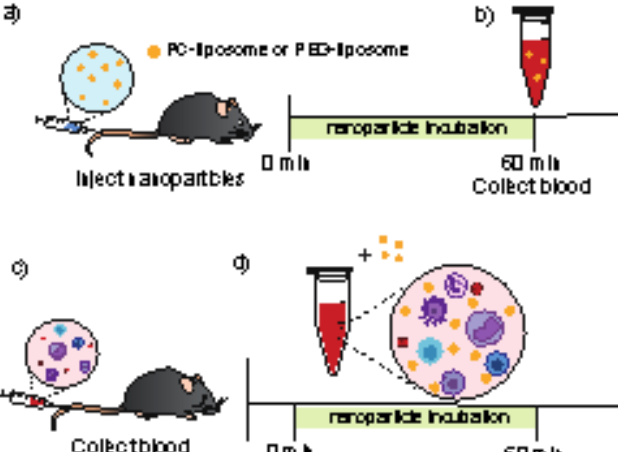

Ф) Colket biood

Add i alopartteles

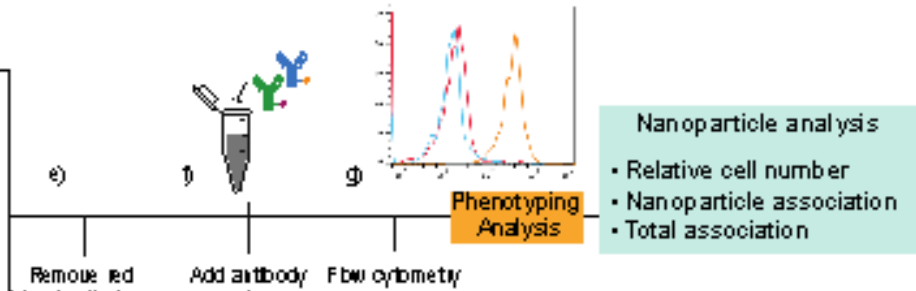

Ex vivo assay

Figure 1. Flow diagram of the Nanoparticle In Vivo Immune- cell Association (NIVIA) assay and ex vivo blood assay. a) In the NIVIA assay, nanoparticles are injected into the tail vein of mice. b) After 1 hour, $100 \mu$ l of blood (containing nanoparticles) is taken from the mice via a cheek bleed. c) For ex vivo blood association, blood is collected from the mouse, and then d) nanoparticles are mixed with the blood for 1 hour. e) For both assays after the 1 hour incubation, red blood cells are removed by lysis. f) Different populations of white blood cells are identified by adding a phenotyping cocktail of antibodies. g) Finally, cells are analysis using flow cytometry.

Association of liposomes to a minor blood subset (such as NK cells) will have less impact on blood clearance than the equivalent association with a major blood subset (such as B cells). Therefore, to determine which immune cell subsets significantly influence clearance of liposome from the blood, we calculated a parameter: 'total association'. The 'total association' of each immune cell subset was calculated by multiplying the number of cells in each subset (relative to the number of T cells) by the background- subtracted MFI.

T cells were chosen as a reference cell population as the proportion of T cells in the CD45+ population (excluding monocytes and neutrophils) remained unchanged in vivo following intravenous injection (Figure S3b). Neutrophils and monocytes were excluded as they share complex relationship with each other in regulating the immune system. ${ }^{[18]}$ Detailed interactions of the different immune cell This article is protected by copyright. All rights reserved. 


\section{WILEY-VCH}

subsets with PC-liposomes and PEG-liposomes are detailed in Figures 2 and 3 respectively. A simplified overview of the total association is summarized in Figure 4.

\section{Association of PC liposomes with immune cells in blood}

PC liposomes showed high association with monocytes both in vivo and ex vivo in whole blood

(Figure 2a-b), however they showed lower association to monocytes in vivo compared to ex vivo, with 1.8x lower mean fluorescent intensity $(M F I)(p<0.001)$ (Figure $2 \mathrm{c})$. The ratio of monocytes:T cells decreased $1 \mathrm{~h}$ after the in vivo administration of PC liposomes (Figure 2d), although the significance $(p=0.1)$ was greater than the confidence limit. This phenomenon was also observed with the injection of PBS only (Figure S4a). The reduced number of monocytes, combined with the decreased number of PC liposomes per cell resulted in 5x lower total association of PC liposomes with monocytes in vivo compared to ex vivo (Figure 2e). 


\section{WILEY-VCH}

a)

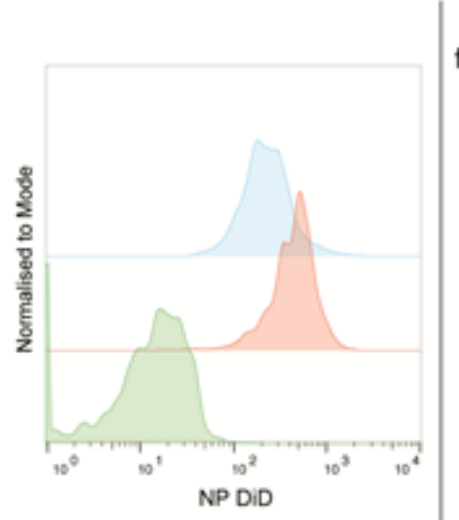

b)

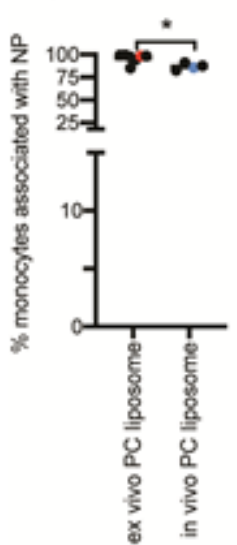

d)

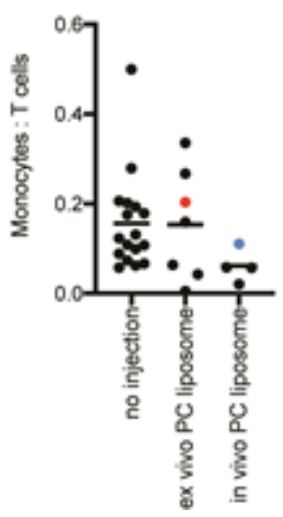

c)

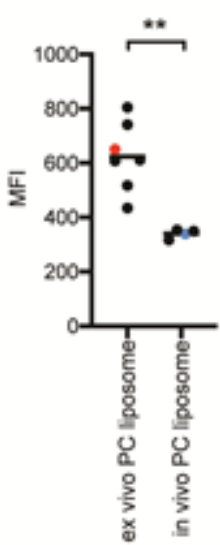

e)

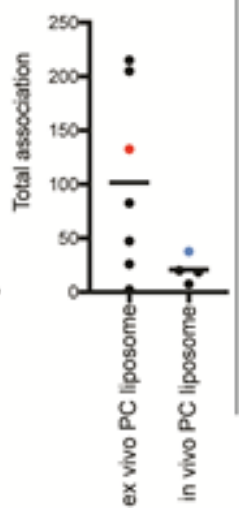

f)

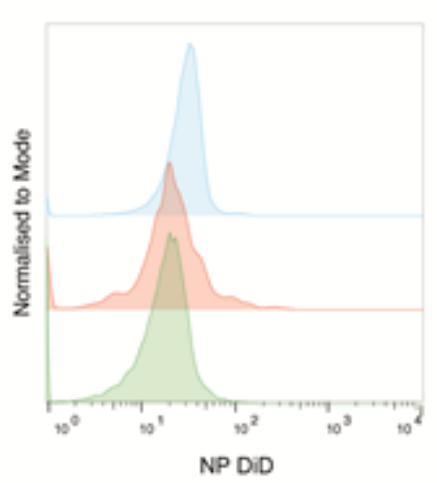

g)

h)
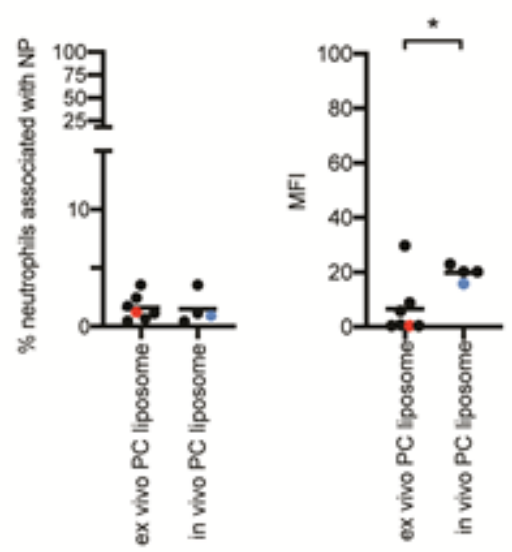

i)

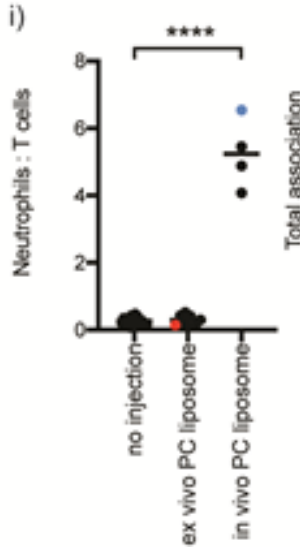

k)

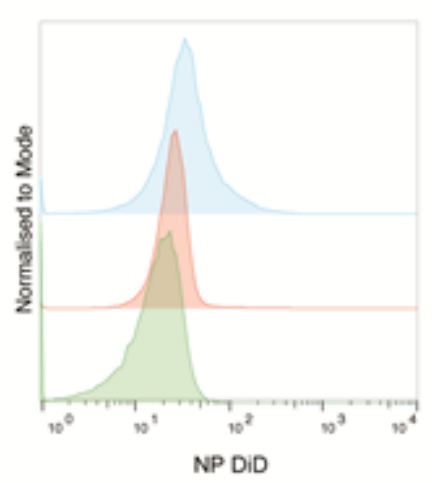

m)
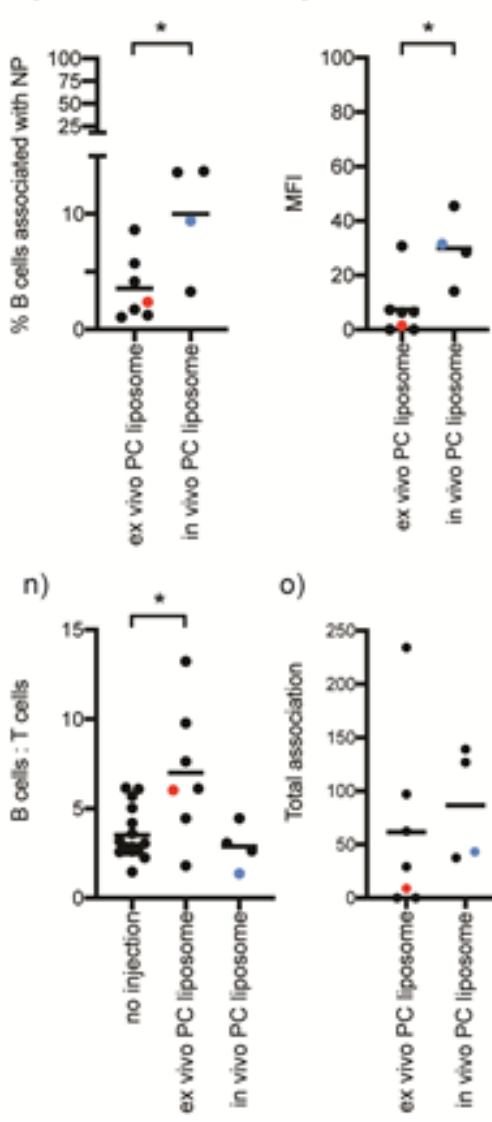

o)

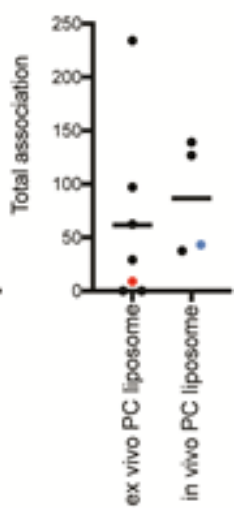

Figure 2. Association of PC liposomes with (a-e) monocytes, $(f-j)$ neutrophils and $(k-0) B$ cells. $(a, f, k)$

Representative overlay histograms of control (green), ex vivo (red) and in vivo (blue) experiments.

$(b, g, l)$ Percentage of NP+ cells. (c, h, m) Corrected geometric mean fluorescent intensity (MFI) of sample minus the average MFI of the PBS. (d, $i, n)$ Proportion of cells to T cells before and after administration of liposomes. $(e, j, o)$ Total association of nanoparticles associated with the cells 


\section{WILEY-VCH}

\section{Association of PEG-liposomes with immune cells in blood}

The same in vivo and ex vivo experiments were also performed with PEG-liposomes. As observed

with PC liposomes, PEG-liposomes showed high levels of association with monocytes (Figure 3a-b).

Similar to the trend observed for PC liposomes, the number of PEG-liposomes associated per

monocyte was $2.5 \mathrm{x}$ lower in vivo than ex vivo (Figure $3 \mathrm{c}$ ). There was a more pronounced decrease in the monocytes:T cell ratio after in vivo administration of PEG-liposomes compared to PC liposomes ( $p<0.05$, Figure $3 d$ vs Figure $2 d$ ). The reduced number of circulating monocytes combined with lower PEG-liposome association per monocyte resulted in 90x lower total association of PEGliposomes in vivo compared to ex vivo. Consequently, the total association of PEG-liposomes to monocytes was 20x lower than PC liposomes in vivo $(\mathrm{p}<0.05$, Figure $3 \mathrm{e}$ vs Figure $2 \mathrm{e})$. 


\section{WILEY-VCH}

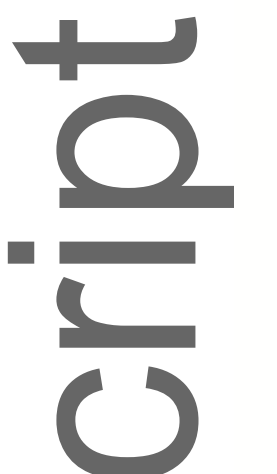

a)

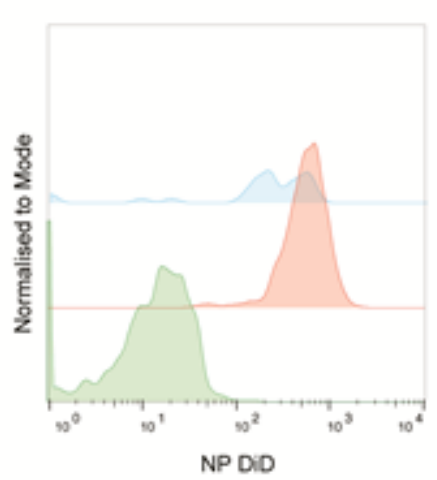

b)

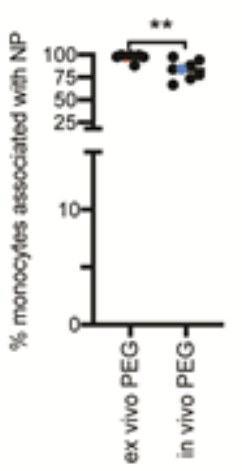

c)
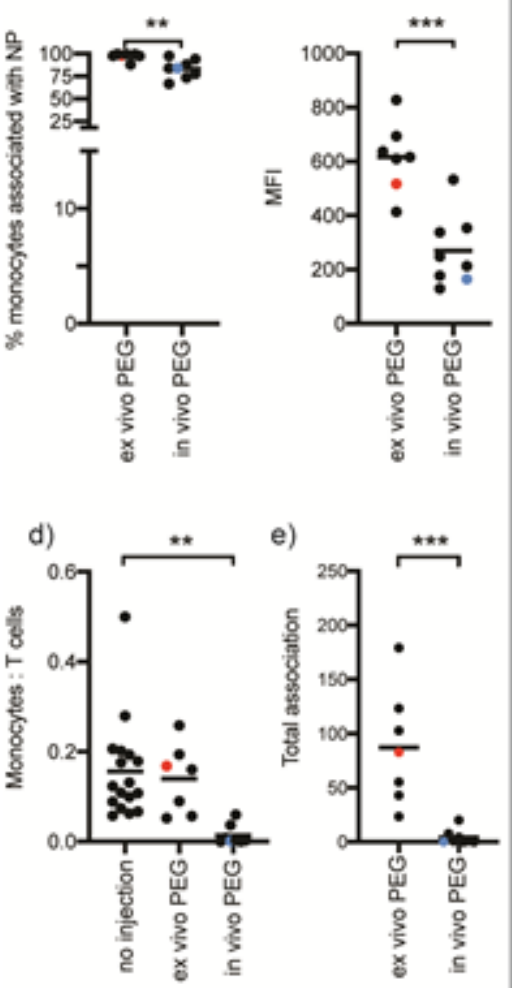

f)

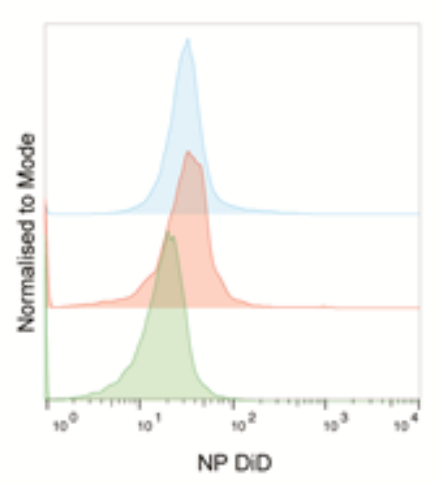

g)

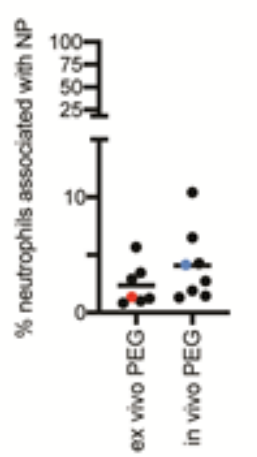

h)
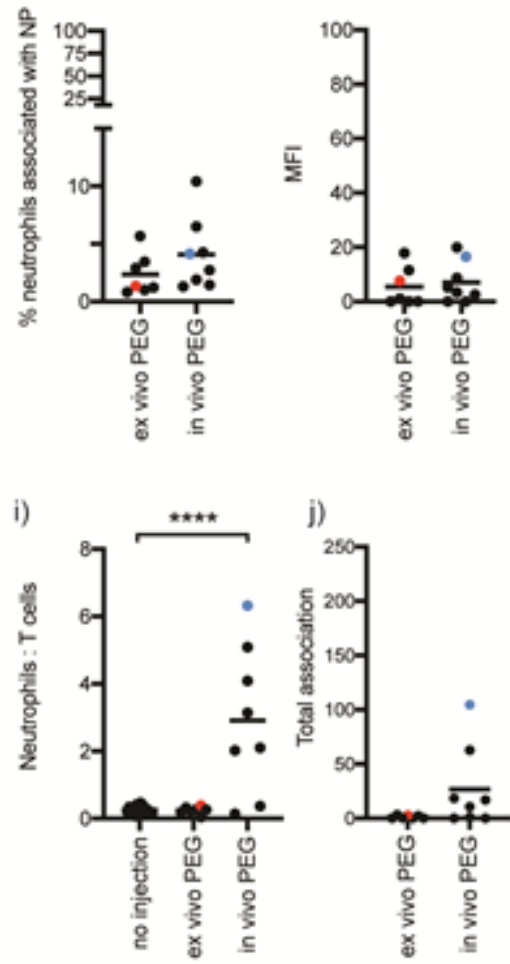

k)

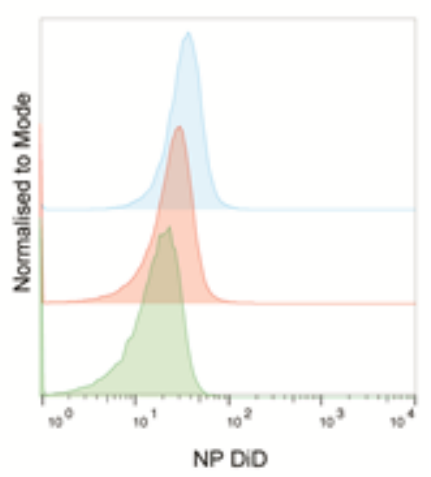

m)

I) n)

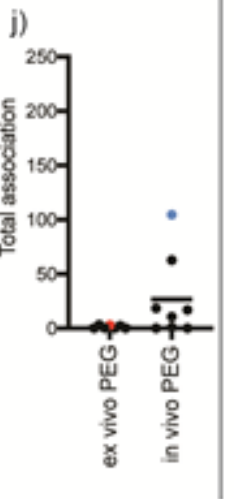

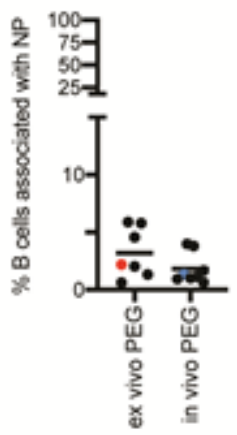

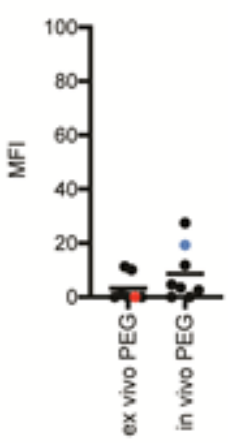

o)

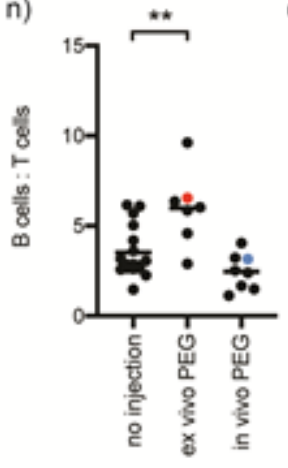

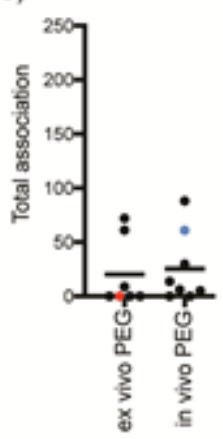

Figure 3. Association of PEGylated liposomes with (a-e) monocytes, ( $f-j)$ neutrophils and ( $k-0) B$ cells. (a, f, k) Representative overlay histograms of control (green), ex vivo (red) and in vivo (blue) experiments. $(b, g, l)$ Percentage of NP+ cells. $(c, h, m)$ Corrected geometric mean fluorescent intensity (MFI) of sample minus the average MFI of the PBS. (d, i, n) Proportion of cells to T cells before and after administration of substances. $(e, j, o)$ Total association of nanoparticles associated with the cells between ex vivo and in vivo experiments. Bars depict mean values. ${ }^{*}=p<0.05,{ }^{* *}=$ $p<0.01, * * *=p<0.001, * * * *=p<0.0001$

This article is protected by copyright. All rights reserved. 


\section{WILEY-VCH}

Similar to PC liposomes, at a cellular level, PEG-liposomes showed significantly lower association to neutrophils than monocytes both in vivo and ex vivo $(\mathrm{p}<0.0001$, Figure $3 \mathrm{f}-\mathrm{h})$. The number of neutrophils also increased following in vivo administration (Figure 3i), however the increase was less than observed for PC liposomes ( $2 x$ lower, Figure 2i) and the PBS control group (2x lower, Figure S4). Compared to PC liposomes, the total association of PEG-liposomes with neutrophils in vivo was 4x lower $(\mathrm{p}<0.01$, Figure $3 \mathrm{j}$ vs Figure $2 \mathrm{j})$.

The association of PEG-liposomes with B cells was similar in vivo and ex vivo (Figure 3k-m), in contrast to PC liposomes where association was significantly higher in vivo (Figure $3 \mathrm{~m}$ vs Figure $2 \mathrm{~m}$ ). The number of B cells was similar after injection (Figure 3n), and the total association of PEGliposomes with B cells was similar in vivo and ex vivo (Figure 3o). Total association of PEGliposomes with B cells in vivo was $3 \mathrm{x}$ lower than PC-liposomes. The total association of PEGliposome to T cells, NK cells and eosinophils was also low in vivo (Figure S6).

\section{Discussion}

Blood is the first point of contact when nanoparticles are administered intravenously. The complex and dynamic nature of the in vivo environment means simple in vitro studies, such as incubating nanoparticles with primary monocytes and macrophages, only give limited insight into the interactions that can occur. ${ }^{[19]}$ Currently, the most robust way to evaluate bio-nano interactions with blood is to use an ex vivo whole blood assay. ${ }^{[12]}$ However in vivo, nanoparticles are subjected to a range of additional cellular and non-cellular factors, such as complex mixing as blood moves through the vasculature, cell-cell/ cell-extracellular matrix interactions, and changes in blood immune cell This article is protected by copyright. All rights reserved. 


\section{WILEY-VCH}

homeostasis, which can all influence how immune cells interact with the nanoparticles. Ex vivo and in vitro assays cannot take into account these environmental factors. To this end, we have developed a method to quantify nanoparticle association with immune cells in blood in vivo, and compared the patterns of association with ex vivo association using whole blood.

In this study, we selected PC and PEG-liposomes which are similar in size and charge to the clinical formulations (without doxorubicin) of Myocet and Doxil respectively. ${ }^{[20]}$ Doxil is a widely used formulation of doxorubicin to treat solid and hematological cancers. ${ }^{[21]}$ Doxil presents a lower clearance rate and longer half-life $(55 \mathrm{~h})$ compared to free doxorubicin $(<1 \mathrm{~h})$ and the analogous nonpegylated liposome formulation, Myocet $(2-3 \mathrm{~h}) .{ }^{[22,23]}$ The increased half-life of Doxil causes side effects such as skin reactions and hypersensitivity, hence, for some cancers (e.g. metastatic breast cancer), Myocet is preferred as minimizes these side effects. ${ }^{[24]}$

Significant differences were observed with the association of liposomes to monocytes in vivo and ex vivo. For both PC liposomes and PEG-liposomes, the MFI of monocytes (and thus the number of nanoparticles per monocyte) in vivo was less than half the signal observed ex vivo. Furthermore, there was a two-fold reduction in circulating monocytes after intravenous administration of PC liposomes or the PBS control. PEG-liposomes caused an even greater 40- fold decrease in the number of circulating monocytes. The decrease in number of circulating monocytes may be due to adherence of the monocytes to the endothelium, where the cells undergo a phenotypic change and extravasate to the tissues. $^{[25]}$ The combination of lower number of liposomes per monocyte, and lower number of monocytes in circulation means that for PEG liposomes the total association to monocytes in vivo was less than $2 \%$ of the total association observed ex vivo and for PC-liposomes it was $20 \%$. This trend is highlighted in Figure 4, which compares the total association of liposomes to the different cell populations, and highlights the differences observed in the NIVIA and ex vivo assays. The reduced 
total liposome association with monocytes in vivo suggests that the ex vivo assay over-estimates the role that monocytes have on liposome clearance.

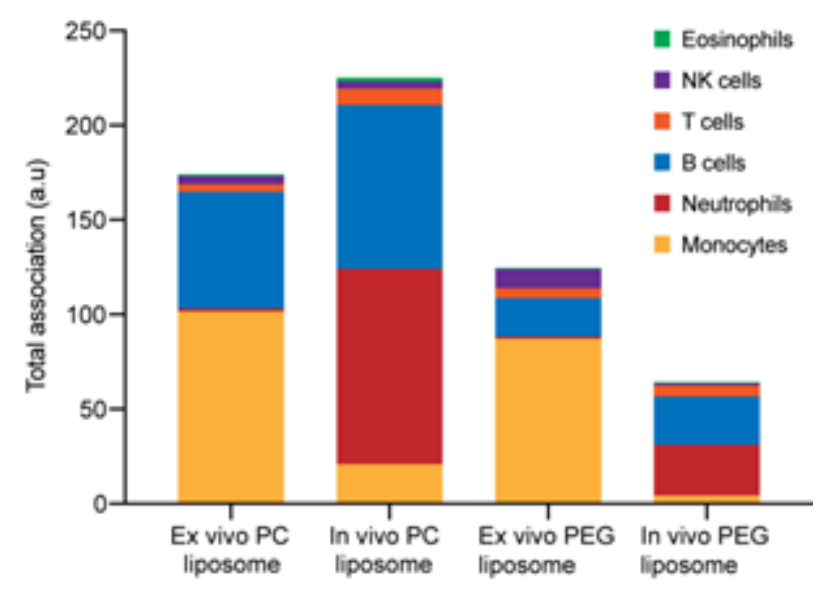

Figure 4. Summary of mean total association of liposomes with each immune cell subset.

Conversely, the increased total association with neutrophils in vivo was underestimated in the ex vivo assay. Although the number of liposomes per neutrophil were significantly lower than the number of liposomes per monocyte (both ex vivo and in vivo), a 10-20 fold increase in the number of neutrophils after intravenous injection altered the total liposome association with neutrophils substantially, to become more significant than the total association with monocytes. Association of PEG-liposomes with neutrophils in vivo accounted for $40 \%$ of the total association with blood cells, compared to $1 \%$ ex vivo. Similarly, association of PC liposomes with neutrophils in vivo account for $45 \%$ of the total association with blood cells, compared to $<1 \%$ ex vivo (Figure 4). Neutrophils in blood are a transient population and represent only $1-2 \%$ of the total pool of cells available in mice. ${ }^{[26]}$ The remaining neutrophils are stored within the bone marrow. ${ }^{[27,28]}$ Although neutrophils are more abundant in human blood compared to mice, the vast majority of these cells are stored in the bone marrow. ${ }^{[2]}$ Neutrophils have a circulation half-life of 6-12 h. ${ }^{[30-32]}$ This short half-life and the capacity to rapidly mobilize cells from the bone marrow enables rapid changes in concentration in blood. Injection of This article is protected by copyright. All rights reserved. 


\section{WILEY-VCH}

nanoparticles into mice and, to a lesser extent PBS, caused a large influx of neutrophils into blood, and the sequestration of $65 \mathrm{x}$ more PC liposomes and 20x PEG-liposomes than would be suggested by the ex vivo assays. The NIVIA assay demonstrated the potentially significant role that neutrophils can play in liposome clearance in vivo, which is not possible to observe with ex vivo assays.

Although the change in balance of monocyte and neutrophil in peripheral blood is consistent with previous septic studies that investigated infection and inflammation, ${ }^{[33-35]}$ endotoxin levels in the PC liposomes, PEG-liposomes and PBS were below immunostimulatory levels. We speculate that the observed change in homeostasis may have been a stress response associated with handling of mice for injection. ${ }^{[36,37]}$ This may have triggered the extravasation of monocytes from blood and recruitment of neutrophils into blood ${ }^{[38,39]}$, altering the homeostasis of monocytes and neutrophils in vivo. External factors such as stress that alter blood homeostasis will also be common in clinical settings.

B cells are a dynamic population that naturally recirculate between the lymph and blood. ${ }^{[40]} \mathrm{We}$ did not observe a large change in the proportion of B cells, suggesting the equilibrium between lymph and blood was not affected by either the intravenous injection or the presence of liposomes. Although the number of particles associating per cell was relatively low, by virtue of their abundance, B cells contributed substantially to nanoparticle sequestration. The association of PC liposomes was slightly higher in vivo compared to ex vivo as indicated by the MFI, however the total association to B cells in vivo and ex vivo was not significantly different for both types of particles. The association of PC liposomes with B cells is consistent with studies involving other particle systems. ${ }^{[32,41]}$ Ex vivo, association of PC liposomes with monocytes and B cells were the dominant interactions, however in vivo, association with neutrophils and B cells were dominant. 


\section{WILEY-VCH}

In the NIVIA assay, liposomes in circulation will be exposed to phagocytic cells within the sinusoidal endothelium in the liver and spleen, which can remove liposomes from circulation. The Kupffer cells in the liver and red pulp macrophages in the spleen are more abundant than blood monocytes and are effective at removing nanoparticles from blood. Moreover, other cells such as the liver sinusoidal endothelial cells can also remove large numbers of nanoparticles from circulation. Therefore, we would anticipate that total association of liposomes with blood cells in vivo would be lower than ex vivo, due to sequestration of nanoparticles in these other organs. Figure 4 shows this trend holds for PEG liposomes, however for PC liposomes, total association was higher in vivo. The increased total association for PC liposomes in vivo can be accounted by the significant increase in the number of circulating neutrophils, and if neutrophils are excluded, total association of PC liposomes in vivo is $\sim 30 \%$ lower than ex vivo.

PEG-liposomes showed 3 to $5 \mathrm{x}$ lower total association compared to PC liposomes for phagocytes (monocytes, neutrophils and eosinophils) and lymphocytes (B cells and NK cells). This demonstrates that as expected, PEGylation of liposomes is able to mask detection from these immune cells to some extent in vivo. ${ }^{[42,43]}$

Human and mouse blood is composed of the same types of cells, however there are differences in the relative proportions of each subset and minor variations in the phenotypes of cells. ${ }^{[44]}$ Neutrophils are the most abundant subset in humans, while in mice, lymphocytes are more abundant. ${ }^{[27]}$ Despite these differences, there is a high level of genetic conservation between the species, so the behavior of individual subsets can be inferred from mice to humans. ${ }^{[44]}$ Ex vivo analysis of nanoparticle-immune cell association enables interactions with human cells to be directly investigated. However, as highlighted in this paper, only the in vivo assay can account for myriad factors that can influence bionano interactions in vivo.

This article is protected by copyright. All rights reserved. 


\section{Conclusion}

In summary, we developed an in vivo assay that provides new insights into the interactions of nanoparticles with immune cells. We found that existing ex vivo blood assays can underestimate the role of neutrophils in liposome clearance, with neutrophils showing $>50$ times higher total association of liposomes in vivo compared to ex vivo. In vivo, the major factor contributing to significantly higher total association with neutrophils was an increase in the number of circulating neutrophils. The increased role of neutrophils observed in vivo with the NIVIA assay is likely to be relevant for a range of other nanoparticles, such as synthetic/natural polymer-based systems and inorganic particles. Furthermore, while monocytes had a higher number of liposomes bound per cell than any other cell type, in vivo we observed both a decrease in the number of monocytes in circulation, as well as a decrease in number of liposomes associated per monocyte. This led to significantly lower total association to monocytes in vivo than ex vivo. While monocytes and B cells play a dominant role in nanoparticle interactions ex vivo, interaction with neutrophils and B cells dominate in vivo.

The in vivo environment has mechanical, biological and structural elements that cannot be faithfully replicated ex vivo. When the full complexity of these factors are taken into consideration more reliable predictions of in vivo nanoparticle behavior can be made. Ex vivo methods of characterizing novel nanoparticles do not give a complete picture of the complex in vivo nanoparticle-immune cell interactions. The NIVIA assay offers a valuable new tool to characterize a wide range of nanoparticles and gives detailed information about their interactions with immune cells. The information gained from the NIVIA assay significantly improves our understanding of nanoparticle interactions with the 


\section{WILEY-VCH}

biological environment and has the potential to be an essential preclinical test for characterizing the in vivo behavior of novel nanoparticles.

\section{Materials and Experimental Methods}

\section{Materials}

DBCO reactive fluorescent liposomes with DiD dye were purchased from Encapsula NanoScience LLC (TN, USA). The product numbers of PEG-liposomes and PC-liposomes were IMF321 and IMF3261 respectively. They were received at stock concentration of $15.98 \mathrm{mg} / \mathrm{ml}$ for PEG liposomes and $14.84 \mathrm{mg} / \mathrm{ml}$ for PC-liposomes. The composition of PEG-liposomes were $57 \mathrm{~mol} \%$ hydrogenated soy phosphatidylcholine, $38 \mathrm{~mol} \%$ cholesterol and $5 \mathrm{~mol} \%$ DSPE-PEG(2000) while PC-liposomes were $68.5 \mathrm{~mol} \%$ L-alpha-Phosphatidylcholine, $30 \mathrm{~mol} \%$ cholesterol and $1.5 \mathrm{~mol} \%$ DBCO PE. The antibodies used in the cocktail to phenotype immune cell subsets were purchased from BD Biosciences (USA).

\section{Liposome characterization}

Stock unmodified and PEG-liposomes were diluted in PBS at a dilution of 1 in 50000 and particle size was measured by Nanoparticle Tracking Analysis software using NanoSight NS3000 System (Malvern Technologies, Malvern, UK). Each liposome was analyzed under constant flow conditions (flow rate $=40$ ) and $5 \times 60 \mathrm{~s}$ videos were captured with camera level of 13. Data was analyzed with NTA 3.4 software with detection threshold of 8. Liposome size distribution was determined by D10, D50 and D90 measurements from the NanoSight. Stock liposomes were diluted 1 in 50000 with 10 $\mathrm{mM}$ phosphate buffer $(\mathrm{pH} 7)$ and the zeta potential of PC-liposomes and PEG-liposomes were 


\section{WILEY-VCH}

measured thrice with Zetasizer Nano ZS (Nano ZS, Malvern, UK) using disposable folded capillary zeta cell.

\section{Fluorescence measurements}

The fluorescence intensities of DiD in the unmodified and PEG-liposomes were measured with spectroscopy (RF-5301PC, Shimadzu). The wavelength was excited at $646 \mathrm{~nm}$ and emitted at the range from $656 \mathrm{~nm}$ to $700 \mathrm{~nm}$ with $1 \mathrm{~nm}$ sampling interval. As the fluorescence intensity of PEGliposomes was 59\% higher than unmodified liposomes, the MFI of unmodified liposomes experiments were multiplied by 1.59 .

\section{In vivo blood studies}

Adult C57BL/6 mice were purchased from Monash Animal Research Platform (MARP, Clayton). All experiments were approved by the Monash Institute of Pharmaceutical Sciences Animal Ethics Committee. Mice $(18 \mathrm{~g}-25 \mathrm{~g})$ were injected intravenously at a dose of $7 \mathrm{mg} / \mathrm{kg}$ with either PEGylated $(n=8)$ or uncoated $(n=4)$ liposomes (equivalent of clinical dose of $20 \mathrm{mg} / \mathrm{m}^{2}$ of Doxorubicin based on body surface area ${ }^{[53]}$ ) (Encapsula NanoScience ImmunoFluor-DBCO, IMF321 [PEG-liposome], IMF3261 [PC-liposome]) in $200 \mu \mathrm{L}$ PBS and blood was collected into a $\mathrm{K}_{2}$ EDTAcoated microtainer (BD Biosciences) after $1 \mathrm{~h}$. A $1 \mathrm{~h}$ timepoint was considered optimal to observe differences in any immune cell association for PC- and PEG- liposomes, consistent with particle halflife's, $3 \mathrm{~h}$ and $55 \mathrm{~h}$, respectively. $200 \mu \mathrm{L}$ of Phosphate-Buffered Saline (PBS, Gibco) was injected intravenously in the control group $(\mathrm{n}=5)$.

The in vivo liposome dose was chosen based on the therapeutic dose of $2 \mathrm{mg} / \mathrm{mL}$ Doxil used clinically (20 mg/m $\mathrm{m}^{2}$ intravenously injected) ${ }^{[24,25]}$ Assuming that the average surface area:weight ratio of a This article is protected by copyright. All rights reserved. 


\section{WILEY-VCH}

mouse is $0.35 \mathrm{~m}^{2} / \mathrm{kg}$, the final dose concentration of liposome used was $7 \mathrm{mg} / \mathrm{kg}{ }^{[31]}$ The final dose volume of PEG-liposomes was multiplied by 0.93 so that the amount of liposomes injected was similar to PC liposomes. The equivalent ex vivo dose was calculated based on a total blood volume of

$7 \%$ of the mouse body weight. ${ }^{[26]}$ The level of endotoxin in both liposome formulations was $0.8-1$ $\mathrm{EU} / \mathrm{mL}$ as from manufacturer and not expected to cause an immune activation.

\section{Ex vivo blood studies}

Mice were weighed to estimate the total blood volume. The total blood volume was assumed to be $7 \%$ of total body weight of the mouse $(70 \mathrm{~mL} / \mathrm{kg}$ ) and the dose equivalent for $100 \mu \mathrm{L}$ of blood collected was calculated. Whole blood was collected from adult C57BL/6 mice via cardiac puncture using a 27 $\mathrm{g}$ needle into $\mathrm{K}_{2}$ EDTA coated microtainer (BD Biosciences). The blood was pre-heated in a thermomixer for 5 mins at $37^{\circ} \mathrm{C}$. PEG- $(n=7)$ or PC- liposomes $(n=7)$ or PBS $(n=5)$ were added to the blood and incubated for $1 \mathrm{~h}$ at $37^{\circ} \mathrm{C}$. Samples were vortexed gently for $1 \mathrm{sec}$ at $15 \mathrm{~min}$ intervals.

\section{Immunophenotyping and Blood processing}

To identify immune cells that are interacting with the DiD- labeled nanoparticles in the blood, freshly collected whole blood was labelled with a cocktail of antibodies (Table S1), including Brilliant Stain buffer (BD) (1:2 dilution) and Fc blocker (1: 100 dilution) for $1 \mathrm{~h}$ at room temperature. Erythrocytes in the blood were then lysed with ACK lysis buffer (Gibco) for 5 min at room temperature. Hanks' Balanced Salt Solution (HBSS) was added to stop the lysis and centrifuged at $500 \mathrm{xg}$ for $7 \mathrm{~min}$. The pellet was washed twice with 1\% PBS/EDTA (pH 7.4) and acquired with a Stratedigm S1000EXi flow cytometer. 


\section{WILEY-VCH}

\section{Flow cytometry}

Bio-nano interactions were analyzed and quantified using FlowJo v10.5.3. Compensation for spectral overlap of fluorochrome-conjugated antibodies was calculated from single stained controls prepared with ultraComp eBeads (ThermoFisher Scientific) according to manufacturer's instructions. The immunophenotyping gating strategy is outlined in Figure S2. As the frequency of dendritic cells was low, dendritic cells were excluded from the analysis. The mean fluorescent intensity (MFI) of liposome fluorescence, DiD was derived from the geometric mean of $\mathrm{DiD}$ in each cell population. To adjust for difference in fluorescence between the two types of liposomes as measured by spectroscopy (RF-5301PC, Shimadzu, activation at $663 \mathrm{~nm}$ ), the MFI of unmodified liposomes were multiplied by 1.59. The average background fluorescence derived from the PBS controls was subtracted from MFI of each cell population. The autofluorescence of the control cells ex vivo (treated with PBS only) and in vivo (injected with PBS only) were similar, so only the in vivo control samples are shown in the representative histograms.

\section{Statistical analysis}

Statistical tests were performed with Prism 8 (GraphPad Software, La Jolla, USA). All groups were assumed to follow a parametric distribution and unpaired $\mathrm{t}$ tests were performed to determine statistical significance between two groups. A $p<0.05$ was determined to be statistically significant. All data are presented with mean. $* \mathrm{p}<0.05, * * \mathrm{p}<0.01, * * * \mathrm{p}<0.001, * * * * \mathrm{p}<0.0001$.

\section{Supporting Information}

Supporting Information is available from the Wiley Online Library or from the author.

\section{Acknowledgements}

This research was supported by a National Health and Medical Research Council Project Grant (1129672, A.P.R.J.) and Career Development Fellowship (1141551, A.P.R.J.) as well as the Aus-tralian Research Council through the Centre of Excellence in Convergent Bio-Nano Science and Technology (A.P.R.J.). A.P.R.J. is also supported through the Monash University Lar-kin's Fellowship Scheme. 


\section{WILEY-VCH}

Received: ((will be filled in by the editorial staff))

Revised: ((will be filled in by the editorial staff))

Published online: ((will be filled in by the editorial staff))

\section{References}

[1] Y. Barenholz, J Control Release 2012, 160, 117-34.

[2] A. S. Thakor, J. V. Jokerst, P. Ghanouni, J. L. Campbell, E. Mittra, S. S. Gambhir, J Nucl Med

2016, 57, 1833-1837.

[3] A. E. Gregory, R. Titball, D. Williamson, Front Cell Infect Microbiol 2013, 3, 13-13.

[4] J.-F. Jin, L.-L. Zhu, M. Chen, H.-M. Xu, H.-F. Wang, X.-Q. Feng, X.-P. Zhu, Q. Zhou, Patient

Prefer Adherence 2015, 9, 923-942.

[5] V. H. Nguyen, B.-J. Lee, Int J Nanomedicine 2017, 12, 3137-3151.

[6] M. A. Dobrovolskaia, A. K. Patri, J. Zheng, J. D. Clogston, N. Ayub, P. Aggarwal, B. W. Neun, J.

B. Hall, S. E. McNeil, Nanomedicine 2009, 5, 106-17.

[7] G. Maiorano, S. Sabella, B. Sorce, V. Brunetti, M. A. Malvindi, R. Cingolani, P. P. Pompa, ACS

Nano 2010, 4, 7481-91.

[8] L. Guerrini, R. A. Alvarez-Puebla, N. Pazos-Perez, Materials (Basel) 2018, 11,

[9] H. L. Kutscher, G. D. Morse, P. N. Prasad, J. L. Reynolds, Pharm Res 2019, 36, 44-44.

[10] C. Vogt, M. Pernemalm, P. Kohonen, S. Laurent, K. Hultenby, M. Vahter, J. Lehtiö, M. S.

Toprak, B. Fadeel, PLOS ONE 2015, 10, e0129008.

This article is protected by copyright. All rights reserved. 


\section{WILEY-VCH}

[11] N. Oh, J.-H. Park, Int J Nanomedicine 2014, 9 Suppl 1, 51-63.

[12] R. De Rose, A. N. Zelikin, A. P. R. Johnston, A. Sexton, S.-F. Chong, C. Cortez, W. Mulholland, F. Caruso, S. J. Kent, Advanced Materials 2008, 20, 4698-4703.

[13] N. B. Shah, G. M. Vercellotti, J. G. White, A. Fegan, C. R. Wagner, J. C. Bischof, Mol Pharm 2012, 9, 2146-55.

[14] I. Constantinescu, E. Levin, M. Gyongyossy-Issa, Artif Cells Blood Substit Immobil Biotechnol $2003,31,395-424$.

[15] C. A. Fromen, W. J. Kelley, M. B. Fish, R. Adili, J. Noble, M. J. Hoenerhoff, M. Holinstat, O. Eniola-Adefeso, ACS Nano 2017, 11, 10797-10807.

[16] R. Münter, K. Kristensen, D. Pedersbæk, J. B. Larsen, J. B. Simonsen, T. L. Andresen, Nanoscale 2018, 10, 22720-22724.

[17] T. J. H. University, Animal Care and Use Committee, http://web.jhu.edu/animalcare/procedures/mouse.html\#additional, accessed: Oct, 2020.

[18] K. Prame Kumar, A. J. Nicholls, C. H. Y. Wong, Cell Tissue Res 2018, 371, 551-565.

[19] M. Bartneck, H. A. Keul, S. Singh, K. Czaja, J. Bornemann, M. Bockstaller, M. Moeller, G. Zwadlo-Klarwasser, J. Groll, ACS Nano 2010, 4, 3073-3086.

[20] U. Bulbake, S. Doppalapudi, N. Kommineni, W. Khan, Pharmaceutics 2017, 9,

[21] I. Sousa, F. Rodrigues, H. Prazeres, R. T. Lima, P. Soares, Cancer Chemother Pharmacol 2018, $82,741-755$.

[22] A. Gabizon, R. Catane, B. Uziely, B. Kaufman, T. Safra, R. Cohen, F. Martin, A. Huang, Y. Barenholz, Cancer Research 1994, 54, 987.

This article is protected by copyright. All rights reserved. 


\section{WILEY-VCH}

[23] A. A. Gabizon, Cancer Invest 2001, 19, 424-36.

[24] M. E. O'Brien, N. Wigler, M. Inbar, R. Rosso, E. Grischke, A. Santoro, R. Catane, D. G. Kieback, P. Tomczak, S. P. Ackland, F. Orlandi, L. Mellars, L. Alland, C. Tendler, Ann Oncol 2004, 15, 440-9.

[25] A. R. Schenkel, Z. Mamdouh, W. A. Muller, Nat Immunol 2004, 5, 393-400.

[26] C. L. Semerad, F. Liu, A. D. Gregory, K. Stumpf, D. C. Link, Immunity 2002, 17, 413-23.

[27] R. Boxio, C. Bossenmeyer-Pourie, N. Steinckwich, C. Dournon, O. Nusse, J Leukoc Biol 2004, $75,604-11$.

[28] K. E. O'Connell, A. M. Mikkola, A. M. Stepanek, A. Vernet, C. D. Hall, C. C. Sun, E. Yildirim, J. F. Staropoli, J. T. Lee, D. E. Brown, Comp Med 2015, 65, 96-113.

[29] C. Rosales, Frontiers in Physiology 2018, 9,

[30] S. von Vietinghoff, K. Ley, J Immunol 2008, 181, 5183-8.

[31] M. Casanova-Acebes, C. Pitaval, L. A. Weiss, C. Nombela-Arrieta, R. Chèvre, A. G. N, Y. Kunisaki, D. Zhang, N. van Rooijen, L. E. Silberstein, C. Weber, T. Nagasawa, P. S. Frenette, A. Castrillo, A. Hidalgo, Cell 2013, 153, 1025-35.

[32] J. M. Adrover, J. A. Nicolás-Ávila, A. Hidalgo, Trends Immunol 2016, 37, 334-345.

[33] B. Thaler, P. J. Hohensinner, K. A. Krychtiuk, P. Matzneller, L. Koller, M. Brekalo, G. Maurer, K. Huber, M. Zeitlinger, B. Jilma, J. Wojta, W. S. Speidl, Scientific reports 2016, 6, 30162-30162.

[34] G. Fingerle, A. Pforte, B. Passlick, M. Blumenstein, M. Strobel, H. W. Ziegler-Heitbrock, Blood $1993,82,3170-6$.

[35] M. Mandl, S. Schmitz, C. Weber, M. Hristov, PLoS One 2014, 9, e112140-e112140.

This article is protected by copyright. All rights reserved. 


\section{WILEY-VCH}

[36] F. S. Dhabhar, W. B. Malarkey, E. Neri, B. S. McEwen, Psychoneuroendocrinology 2012, 37, $1345-1368$.

[37]

L. M. Ince, J. Weber, C. Scheiermann, Frontiers in Immunology 2019, 9,

[38] C. Auffray, D. Fogg, M. Garfa, G. Elain, O. Join-Lambert, S. Kayal, S. Sarnacki, A. Cumano, G. Lauvau, F. Geissmann, Science 2007, 317, 666-70.

[39] D. Begandt, S. Thome, M. Sperandio, B. Walzog, J Leukoc Biol 2017, 102, 699-709.

[40] J. P. Girard, C. Moussion, R. Förster, Nat Rev Immunol 2012, 12, 762-73.

[41] J. J. Glass, D. Yuen, J. Rae, A. P. R. Johnston, R. G. Parton, S. J. Kent, R. De Rose, Nanoscale 2016, 8, 8255-8265.

[42] Q. Yang, S. W. Jones, C. L. Parker, W. C. Zamboni, J. E. Bear, S. K. Lai, Mol Pharm 2014, 11, $1250-8$.

[43] C. D. Walkey, J. B. Olsen, H. Guo, A. Emili, W. C. Chan, J Am Chem Soc 2012, 134, 2139-47.

[44] J. Mestas, C. C. W. Hughes, The Journal of Immunology 2004, 172, 2731-2738.

This article is protected by copyright. All rights reserved. 


\section{WILEY-VCH}

Y. R. Ong, R. De Rose, A.P.R. Johnston*

In vivo quantification of nanoparticle association with immune cell subsets in blood

Clearance of nanoparticles from circulation in blood is a significant hurdle for the therapeutic application of novel nanomedicines. Here we developed a Nanoparticle In Vivo Immune-cell Association (NIVIA) assay that shows how the behavior of nanoparticles after intravenous injection is influenced by factors unique to the in vivo environment and cannot be replicated ex vivo.
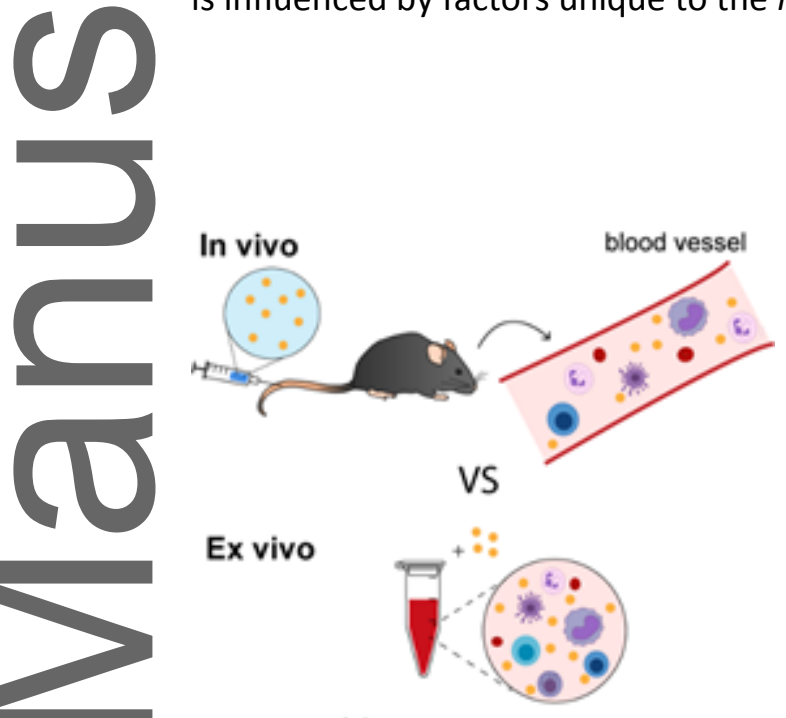

- nanoparticle

This article is protected by copyright. All rights reserved. 


\section{University Library}

\section{- M M N E R VA A gateway to Melbourne's research publications}

Minerva Access is the Institutional Repository of The University of Melbourne

Author/s:

Ong, YR;De Rose, R;Johnston, APR

Title:

In Vivo Quantification of Nanoparticle Association with Immune Cell Subsets in Blood

Date:

2021-02-28

Citation:

Ong, Y. R., De Rose, R. \& Johnston, A. P. R. (2021). In Vivo Quantification of Nanoparticle Association with Immune Cell Subsets in Blood. ADVANCED HEALTHCARE MATERIALS, 10 (9), https://doi.org/10.1002/adhm.202002160.

Persistent Link:

http://hdl.handle.net/11343/297359 